\title{
Design of a High-Power High-Efficiency Multi-Receiver Wireless Power Transfer System
}

\author{
Yuyu Zhu ${ }^{1}$, Hanyu Zhang ${ }^{1}$, Zuming Wang ${ }^{1}$, Xin Cao ${ }^{1, *}$ and Renyin Zhang ${ }^{2}$ \\ 1 School of Information Engineering, Southwest University of Science and Technology, \\ Mianyang 621010, China; zhuyuyu@swust.edu.cn (Y.Z.); izhanghy@hotmail.com (H.Z.); \\ wangzuming1998@163.com (Z.W.) \\ 2 School of National Defence Science and Technology, Southwest University of Science and Technology, \\ Mianyang 621010, China; zhangrenyin1230@163.com \\ * Correspondence: caoxin@swust.edu.cn
}

Citation: Zhu, Y.; Zhang, H.; Wang, Z.; Cao, X.; Zhang, R. Design of a High-Power High-Efficiency Multi-Receiver Wireless Power Transfer System. Electronics 2021, 10, 1308. https://doi.org/10.3390/ electronics10111308

Academic Editor: Francisco G. Montoya

Received: 2 April 2021

Accepted: 28 May 2021

Published: 30 May 2021

Publisher's Note: MDPI stays neutral with regard to jurisdictional claims in published maps and institutional affiliations.

Copyright: (c) 2021 by the authors. Licensee MDPI, Basel, Switzerland. This article is an open access article distributed under the terms and conditions of the Creative Commons Attribution (CC BY) license (https:/ / creativecommons.org/licenses/by/ $4.0 /)$.

\begin{abstract}
This paper proposes a new control method to regulate the power flow into multiple receivers. This system consists of one transmitter controller and three receiver controllers. They work independently to decide the power distribution with their combined operation. The simulated and experimental models have been built, and the experimental results are in good agreement with the theoretical analysis results. The proposed method is robust, flexible, and generalizable, and can be employed under various wireless charging conditions.
\end{abstract}

Keywords: uneven power distribution; competition mechanism; high efficiency; high isolation

\section{Introduction}

Wireless power transfer (WPT) is a technology that enables power transfer in a convenient and safe manner without any physical contact. WPT is widely used in many areas, such as cell phones, household appliances, industrial robots, and electric vehicles. There are many WPT system that can charge multiple receivers with a single transmitter. Tightly coupled inductive technology based on wireless power consortium (WPC or Qi) or loosely coupled magnetic resonant technology based on air fuel has been widely used in multi-receiver WPT systems, where receivers are powered simultaneously.

Many literatures have investigated various aspects regarding multi-receiver WPT systems, such as efficiency optimization [1-5], multi-frequency WPT [6], transfer characteristics [7,8], energy encryption [9], omnidirectional WPT [10], cross coupling effect [11,12], reconfigurable WPT [13], and bidirectional power transfer [14]. To extend the application scope of multi-receiver WPT, the system should be more generalizable and robust with flexible receiver number and power levels.

However, there are some challenges with the power distribution of multi-receiver WPT systems. First, receivers close to the transmitter tend to absorb more power than the ones far away [15], whereas the power demand of each receiver should be decided by its load instead of receiver distance. Second, many factors, such as power demand, receiver distances and receiver number may dynamically change. Third, the power capacity of the transmitter becomes more insufficient to meet the demands with the increase in receiver number. In most cases, the peak power demand is much higher than the average power demand [16]. Therefore, the transmitter may be overloaded if the receivers absorb more power than the capacity of the transmitter.

Recently, some methods have been proposed to control the power distribution in multi-receiver WPT systems. In [16], the frequency is used as the communication channel for the entire system. Receivers are configured to different priority levels. When the transmitter is on the verge of overloading, the power of all the receivers is decreased simultaneously. Reference [17] proposes a power distribution scheme that can be adapted 
to the variations in the load parameters. This scheme employs an impedance regulator at the receiver side to match the power demand of the loads. A transmitter controller is used to alter a capacitor array to control the total equivalent impedance. Reference [18] adopts a time-sharing method, which selectively and exclusively delivers power to only one designated receiver among multiple receivers. The power division ratio is controlled by changing the duration time ratio for power transfer. This method is realized by separating the resonant frequency of every receiver. In [19], multiple frequencies are tuned into one transmitting coil with multiple inverters. Receivers are tuned at different corresponding frequencies; thus, the power transferred to each receiver can be controlled independently. However, this configuration is complex and costly. Reference [15] proposes a method to control the power division ratio through an impedance matching circuit. However, the power division ratio is sensitive to the coupling coefficient, frequency, and load resistance, making this method less flexible. References $[4,20]$ have designed the power division ratio by adjusting the load impedance. However, the load impedance cannot be changed in some practical WPT systems. Reference [21] proposed a game-theory-based power distribution control method, whereby the charging power distribution is determined and updated through the interplay between the existing receivers.

Although several different types of power distribution approaches have proposed in aforementioned literature, these solutions still cannot satisfy all the requirements of large-scale application, such as power demand of each receiver, transmitter overloading prevention, equivalent impedance matching, flexible power ratio, easy configuration, and compact size. Some methods are costly and complicated in hardware and software implementation. In addition, some methods have limitations in the number of receivers.

To improve performance and overcome some limitations of the existing methods, this paper has proposed a new power distribution method for multi-receiver WPT system. This method introduces competition mechanism to distribute power, which can achieve dynamic power distribution. The proposed method is simple in both hardware and software implementation with no limit in the number of receivers.

\section{Multi-Receiver WPT Systems}

\subsection{Competition Theory Analysis}

A typical multi-receiver WPT system is shown in Figure 1. The transmitter is powered by a DC power supply. The current in the transmitting coil produces magnetic field around the transmitting coil, voltage is induced in all receiving coils, and then power will be delivered to all receivers simultaneously. When a receiver needs more power, the transmitter sees impedance changes. Since all receiver coils are parallel, the cross-coupling can usually be neglected [22,23], the reflected impedance $Z_{i}$ can be derived as [23].

$$
Z_{i}=\frac{\omega^{2} M_{(i)}^{2}}{Z_{r(i)}+R_{L(i)}}
$$

where $\omega$ is the operating angular frequency; $M_{(i)}$ is the mutual inductance between the TX coil and the $i$-th RX coil; $Z_{r i}$ is the equivalent impedance seen though RX. Under resonance condition, the load impedance seen by the input is

$$
Z_{\text {in }}=\sum_{i=1}^{n} Z_{i}
$$

The output power from the input is first transferred to the $i$-th RX coil, and then to the load. Then, the power received by the $i$-th load can be calculated to be

$$
P_{o(i)}=P_{i} \frac{\operatorname{Re}\left\{Z_{i}\right\}}{\sum_{i=1}^{n} \operatorname{Re}\left\{Z_{i}\right\}} \cdot \frac{R_{L(i)}}{\operatorname{Re}\left\{Z_{r(i)}\right\}+R_{L(i)}}
$$



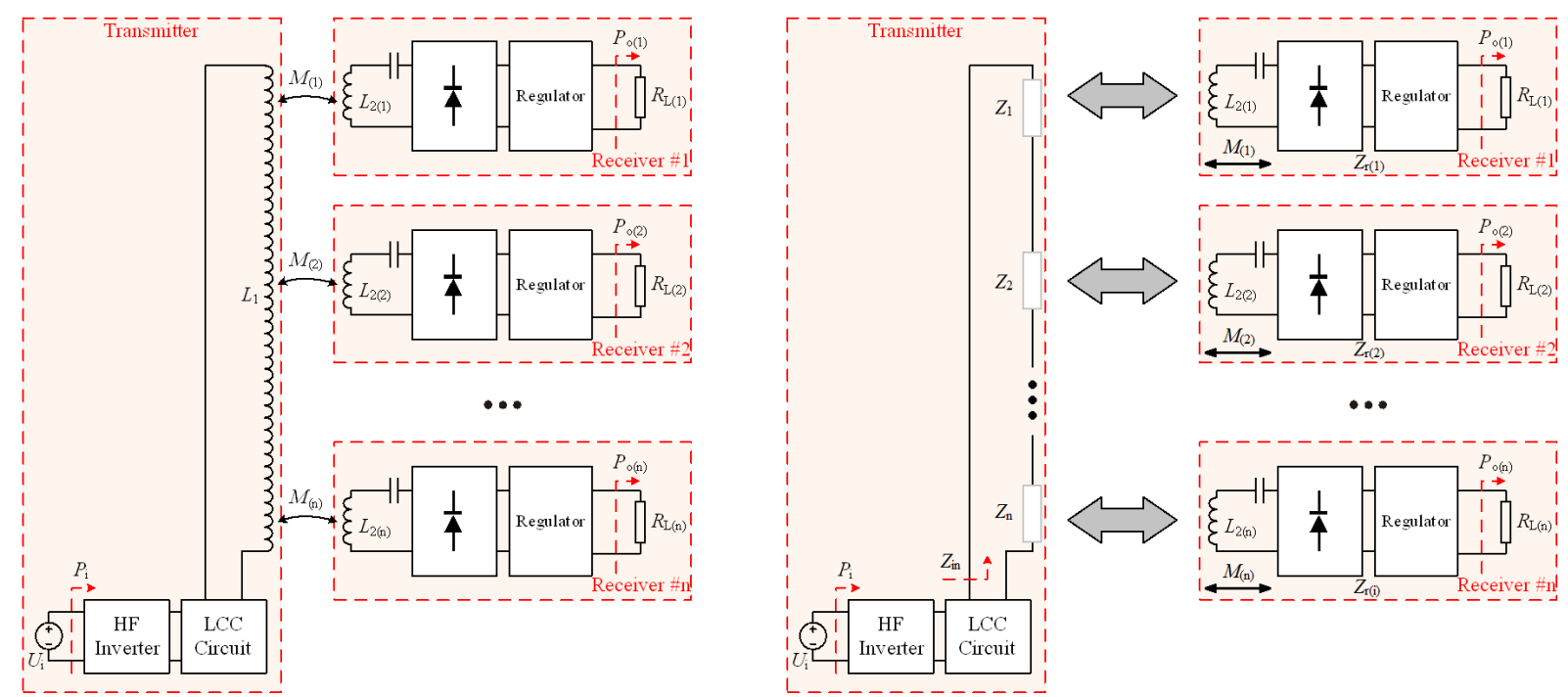

Figure 1. Schematic of the multi-receiver WPT system.

\subsection{Transmitter Analysis}

The schematic of the transmitter is shown in Figure 2. A high-frequency (HF) inverter converts the DC power to HF AC power, and then the power is driven into the transmitting compensation circuit through the coils.

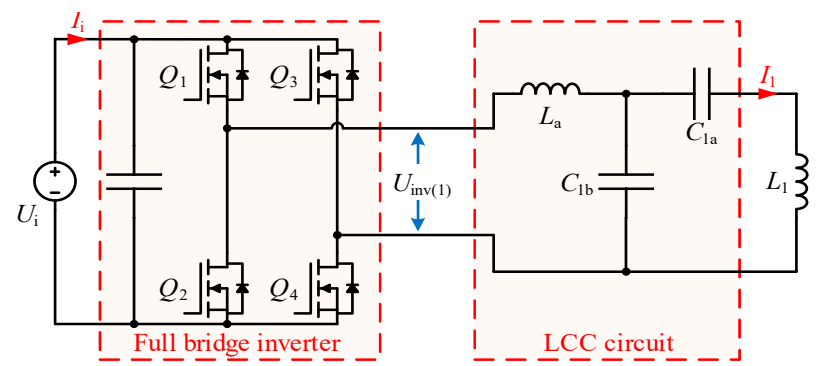

(a)

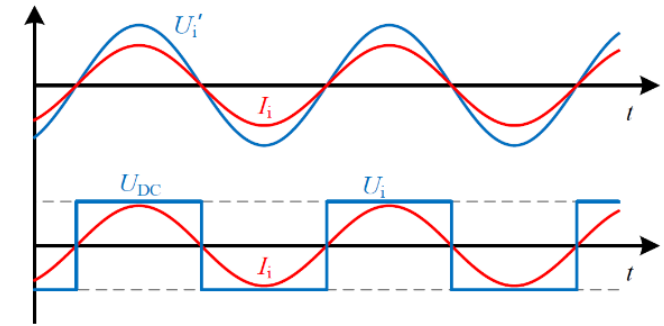

(b)

Figure 2. Schematic of the transmitter. (a) Circuit structure (b) voltage and current waveform.

The inverter adopts a full bridge configuration, and the amplitude of its output is controlled by phase-shift angle $\alpha$. The waveform of inverter output voltage $U_{i n v}$ is a square waveform which consists of an infinite number of harmonics. When the rms of the fundamental component of $U_{i n v}$ (which is defined as $U_{i n v(1)}$ ) is considered, the relationship between $U_{i n v(1)}$ and phase-shift angle $\alpha$ is

$$
U_{i n v(1)}=\frac{2 \sqrt{2}}{\pi} \cos \left(\frac{\alpha}{2}\right) U_{i}
$$

The LCC circuit is employed for the transmitter as the compensation circuit, which consists of three components $L_{a}, C_{1 a}$, and $C_{1 b}$. The matching condition of the LCC circuit is [5]:

$$
\omega L_{a}=\frac{1}{\omega C_{1 b}}=\omega L_{1}-\frac{1}{\omega C_{1 a}}
$$

where $\omega$ is the angular frequency and $L_{1}$ is the self-inductance of transmitting coil. Then, the rms value of transmitting coil current $I_{1}$ is [24]:

$$
I_{1}=\frac{U_{i n v(1)}}{\omega L_{a}}
$$


The transmitting coil current is proportional to $U_{i n v(1)}$, irrespective of the load impedance. Compared with other common compensation circuits such as series compensation circuit, LCC compensation circuit has advantage of constant current characteristics, which is helpful to decouple receivers. The duty cycle is controlled under constant frequency and it is done easily.

\subsection{Receiver Analysis}

The receiver consists of a receiving coil, a compensation circuit, a rectifier, and a regulator, as shown in Figure 3 . The induced voltage $U_{2}$ in receiving coil can be regarded as a voltage source in series with receiver coil, and its rms value can be calculated as:

$$
U_{2}=\omega M I_{1}
$$

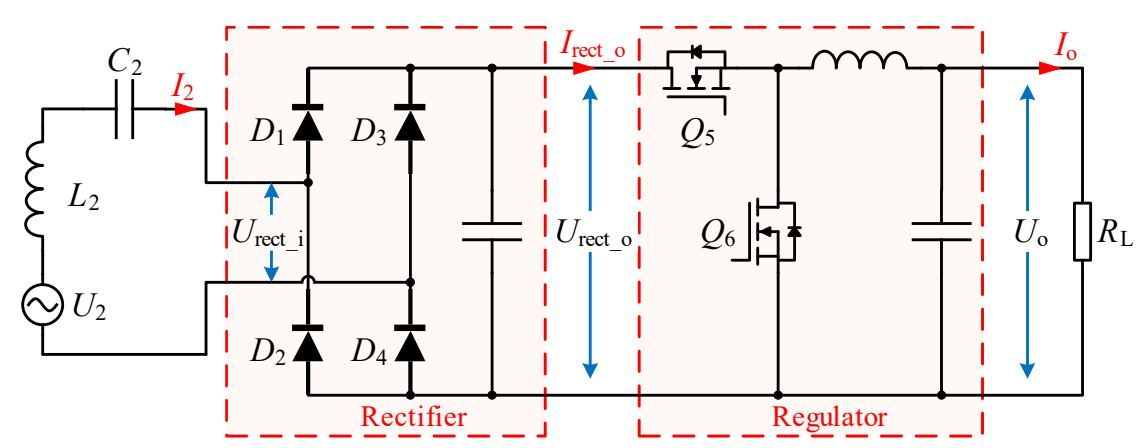

Figure 3. Schematic of the receiver.

The input current of the rectifier $I_{2}$ is distorted because of the non-linear characteristics of the rectifier. The average power supplied by $U_{2}$ is:

$$
P_{2}=U_{2} I_{2}
$$

The rectifier input waveform is shown in Figure 4. The output current of the rectifier can be expressed using Fourier harmonic expansion as [25].

$$
I_{\text {rect_o }}=\sqrt{2} I_{2}\left(\frac{2}{\pi}-\frac{4}{3 \pi} \cos 2 \omega t-\frac{4}{15 \pi} \cos 4 \omega t-\frac{4}{35 \pi} \cos 6 \omega t \ldots\right)
$$
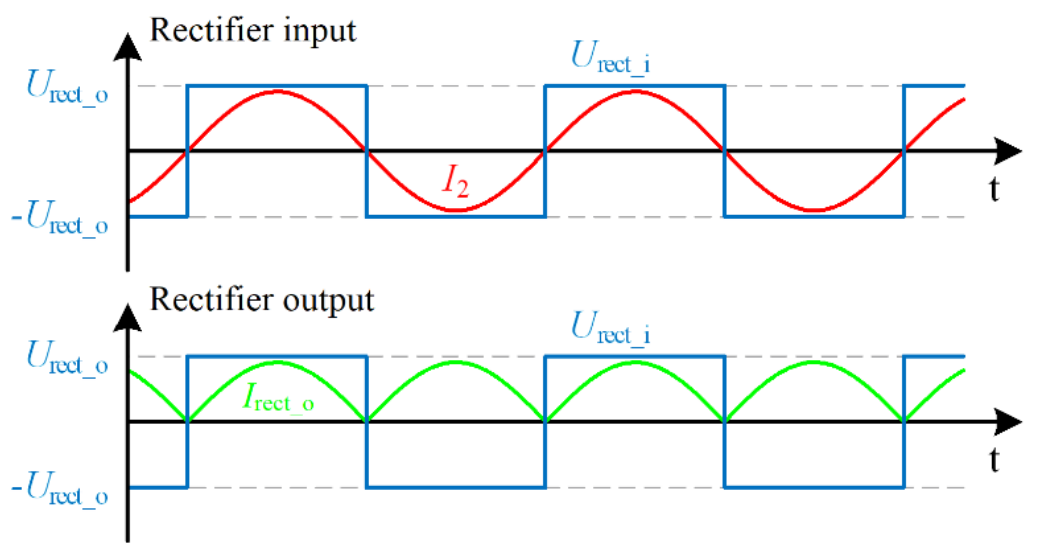

Figure 4. The waveform of the receiver rectifier. 


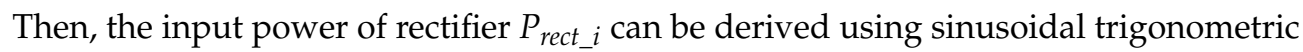
functions calculus [26]. Since the power is calculated within a time period, only the DC term is not zero and $P_{\text {rect } i}$ can be calculated to be:

$$
P_{\text {ret_ } i}=\frac{1}{T} \int_{0}^{T} U_{\text {rect_o }} I_{\text {rect_o }}=\frac{2 \sqrt{2}}{\pi} U_{\text {rect_o }} I_{2}
$$

where $U_{\text {rect_o }}$ is the rectifier output voltage. The power losses of the rectifier is very small and, therefore, can be neglected, and the average power supply can be calculated as:

$$
P_{2} \approx P_{\text {rect_ } i}
$$

The relationship between $U_{2}$ and $U_{\text {rect_oo }}$ can be derived from (10):

$$
U_{\text {rectoo }}=\frac{\pi}{2 \sqrt{2}} U_{2}
$$

The regulator is a half-bridge converter, and its output voltage $U_{o}$ is

$$
U_{o}=D U_{\text {rect_o }}
$$

where $D$ is the duty cycle of the regulator.

By combining (4), (6), (11), (12) and (13), the output power to the load $P_{o}$ can be derived as:

$$
P_{o}=\frac{U_{o}^{2}}{R_{L}}=\frac{\left(M U_{i} D \cos \frac{\alpha}{2}\right)^{2}}{R_{L} L_{a}^{2}}
$$

It can be noted from (14) that $P_{o}$ can be controlled by $\alpha$ and $D$.

In our multi-receiver WPT system, the transmitting LCC circuit could minimize the effect of cross coupling due to its constant current characteristic of the transmitting coil [20]. The output power satisfies (14) and can be rewritten as a function of the control variable $\alpha$ and duty cycle of the ith regulator $D_{(i)}$ :

$$
P_{o(i)}=f\left(\alpha, D_{(i)}\right)=\frac{\left(U_{i} \cos \frac{\alpha}{2}\right)^{2}}{L_{a}^{2}} \times \frac{\left(M_{(i)} D_{(i)}\right)^{2}}{R_{L(i)}}
$$

where $M_{(i)}$ is the mutual inductance between the transmitting coil and the coil of receiver $\# i, R_{L(i)}$ is the load resistance of receiver $\# i$. $P_{o(i)}$ can be regulated by the two parameters indicated in (15): $\alpha$ and $D_{(i)}$. In a single receiver WPT system, only one parameter needs to be changed to achieve desired power regulation, with the other one fixed.

The total output power of the receivers $P_{o_{-} \text {sum }}$ can be expressed as follows:

$$
P_{o_{-} \text {sum }}=\sum_{i=1}^{n} P_{o(i)}=\frac{\left(U_{i} \cos \frac{\alpha}{2}\right)^{2}}{L_{a}^{2}} \times \sum_{i=1}^{n} \frac{\left(M_{(i)} D_{(i)}\right)^{2}}{R_{L(i)}}
$$

where $n$ is the number of receivers.

The efficiency of the entire system $\eta$ is defined as:

$$
\eta=\frac{P_{O_{\_} \text {sum }}}{P_{i}}
$$

where $P_{i}$ is the input power of transmitter, which can be derived as:

$$
P_{i}=\frac{P_{o_{-} \text {sum }}}{\eta}=\frac{\left(U_{i} \cos \frac{\alpha}{2}\right)^{2}}{\eta L_{a}^{2}} \times \sum_{i=1}^{n} \frac{\left(M_{(i)} D_{(i)}\right)^{2}}{R_{L(i)}}
$$


For a multi-receiver WPT system, the power distribution control strategy should satisfy two basic principles. First, the transmitter has limited power capacity, and it should not overload at any given time. Second, the power demand by each receiver should be satisfied as much as possible. When the power capacity of the transmitter meets the demand of all the receivers, every receiver should receive the required amount of power. When the power capacity of transmitter is lower than the total power demand of the receivers, and receivers should absorb less power than their demands to prevent overloading.

\subsection{The Proposed Structure Analysis}

Our proposed structure employs double-side control and it is comprised of two types of controllers. The first type is the transmitter controller. There is only one transmitter controller in the multi-receiver WPT system, and it is located at the transmitting side to regulate the total power of the WPT system and to prevent overloading the DC power supply. The second type is the receiver controller. Every receiver has an individual receiver controller. Based on the circuit topology, the system provides adaptive output based on the load requirement in each receiver. The current in the transmitting coil produces magnetic field around the transmitting coil, and voltage is induced in all receiving coils, and then power is delivered to all receivers simultaneously. The regulators control the output power of each load are based on the design requirements. Power distribution comes from mutual inductions and actual DCs loads.

The structure of the proposed transmitter controller is shown in Figure 5. This system contains a feedback loop with a PI controller and phase-shifted signal generator. The voltage and current values are sampled in to calculate the input power $P_{i}$. This value is subtracted with the instructed power value $P_{i}^{*}$ to obtain the error power $P_{i \_ \text {err }}$. Then the error power is normalized in the domain $(0,1)$ to feedback the phase-shifted signal generator, which controls the gate driver of the HF invertor. Additional anti-trigonometric square root function is integrated to increase the stability and robustness of the controller.

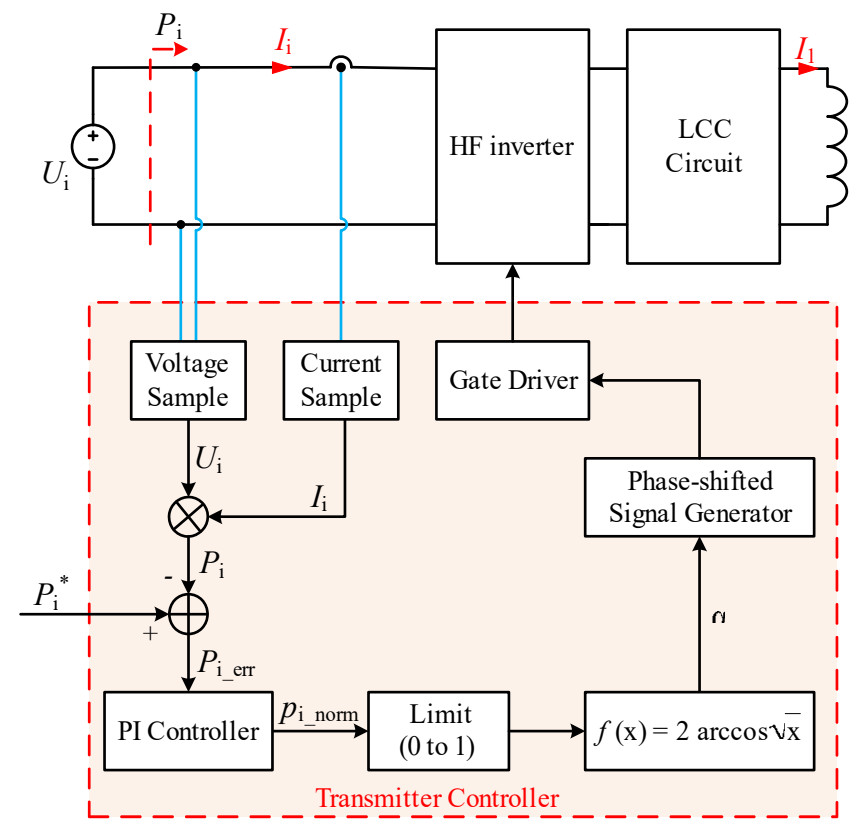

Figure 5. Scheme of transmitter controller.

The transmitter controller would control the power of the entire system such that the instruction input power is not exceeded. The instruction input power is set to be slightly lower than the power capacity of the DC power supply to preserve redundancy power of the supply. First, the input voltage and input current of the inverter are sampled through a sampling circuit, and the input power of the inverter is measured by multiplying the input voltage and $t$ current. The error between the instructed and measured power is calculated 
through subtraction. A proportional-integral (PI) controller then regulates the input power of the inverter based on the input error.

It can be seen from (18) that the DC power supply has the maximum output power $P_{i \_ \text {max }}$ when the inverter phase shift angle $\alpha$ is equal to 0 , that is:

$$
P_{i \_ \text {max }}=\left.P_{i}\right|_{\alpha=0^{\circ}}=\frac{U_{i}^{2}}{\eta L_{a}^{2}} \times \sum_{i=1}^{n} \frac{\left(M_{(i)} D_{(i)}\right)^{2}}{R_{L(i)}}
$$

The output of the PI controller represents the normalized power $p_{i \_n o r m}$, which is the ratio of the $P_{i}$ to $P_{i \_m a x}$. Combining (18) and (19), $p_{i \_n o r m}$ can be derived as:

$$
p_{i_{-} \text {norm }}=\frac{P_{i}}{P_{i_{-} \max }}=\cos ^{2}\left(\frac{\alpha}{2}\right)
$$

Since $p_{i \_n o r m}$ is between 0 and 1 , the output of the PI controller is limited from 0 to 1 . By rewriting (20), the phase shift angle of the inverter $\alpha$ can be derived as:

$$
\alpha=2 \arccos \sqrt{p_{i_{-} \text {norm }}}
$$

Therefore, a math function block is inserted after the PI controller to calculate the phase shift angle $\alpha$. Finally, a phase-shifted signal generator produces the control signal for the inverter, and the gate driver drives the semiconductor switches in the inverter.

The transmitter controller regulates the inverter input power to prevent overloading of the DC power supply. Two possible states may appear for the transmitter controller. In state $1, P_{i}^{*}$ is less than $P_{i \_ \text {max }}$, while in state 2 , the instruction input power $P_{i}^{*}$ is greater than or equal to $P_{i \_ \text {max }}$, as shown in Figure 6.

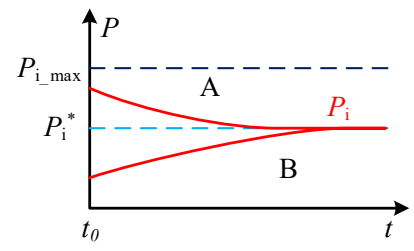

(a)

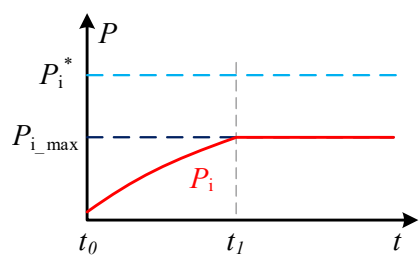

(b)

Figure 6. Working states in transmitter controller. (a) State 1 (b) State 2.

State 1: $P_{i}^{*}<P_{i \_m a x}$. Considering a scenario at time $t_{0}, P_{i}$ is greater than $P_{i}^{*}$, the error power $P_{i \_ \text {err }}$ is negative, the PI controller decreases $P_{i \_n o r m}, \alpha$ increase, and, therefore, $P_{i}$ decreases. On the contrary, if $P_{i}$ is less than $P_{i}^{*}$ at $t_{0}, P_{i \_ \text {err }}$ would be positive, and the PI controller would increase $P_{i \_n o r m}$, and $\alpha$ would decrease, and therefore $P_{i}$ would be increased. The PI controller would adjust its output only if $P_{i}$ is not equal to $P_{i}^{*}$. Finally, in state $1, P_{i}$ will be equal to $P_{i}^{*}$ in the steady state. Moreover, there is no static error due to the integral part in the PI controller.

State 2: $P_{i}^{*} \geq P_{i_{-} \max }$. If $P_{i}$ is less than $P_{i \_ \text {max }}$ at time $t_{0}$, then $P_{i}$ must also be less than $P_{i}^{*}$. Similar to state $1, P_{i \_r r}$ is positive, and the PI controller would try to increase $P_{i \_n o r m}$ to increase $P_{i}$. When $P_{i \_n o r m}$ increases to 1 at $t_{1}, P_{i}$ is equal to $P_{i \_m a x}$ but still less than $P_{i}^{*}$. Therefore, the PI controller tends to continue to increase $P_{i \_n o r m}$. However, $P_{i_{-} \text {norm }}$ is limited to be no greater than 1 , so the $P_{i \_n o r m}$ would remain equal to 1 , then the transmitter controller is saturated. Finally, in the steady state, $P_{i}$ will be equal to $P_{i \_ \text {max }}$ and would not increase to $P_{i}^{*}$. 
In summary, under steady state, $P_{i}$ is always less than $P_{i}^{*}$ and $P_{i \_ \text {max }}$ under the control of the transmitter controller, and can be calculated as

$$
P_{i \_ \text {max }}=\left.P_{i}\right|_{\alpha=0^{\circ}}=\frac{U_{i}^{2}}{\eta L_{a}^{2}} \times \sum_{i=1}^{n} \frac{\left(M_{(i)} D_{(i)}\right)^{2}}{R_{L(i)}}
$$

Each receiver has an individual receiver controller to regulate its output for the load. This receiver controller is designed for constant-voltage output. The structure of the receiver controller is shown in Figure 7. The input to the receiver controller is the instructed output voltage $U_{(o) i}{ }^{*}$. The output voltage $U_{o(i)}$ is sampled through the sampling circuit. The error between $U_{(o) i}{ }^{*}$ and $U_{o(i)}$ is fed into the PI controller, and the output of which is the duty cycle $D_{(i)}$ for the regulator. $D_{(i)}$ is limited between 0 and 1 . The PWM generator would generate the PWM control signal for the regulator, and the semiconductor switches are driven by the gate driver. Noting that once the instructed output voltage $U_{(o) i}{ }^{*}$ is determined, the equivalent instruction output power $P_{o(i)}{ }^{*}$ can be determined by:

$$
P_{o(i)}^{*}=\frac{U_{o(i)}^{*}{ }^{2}}{R_{L(i)}}=\frac{\left(D^{*} U_{r e c t \_o}^{*}\right)^{2}}{R_{L(i)}}
$$

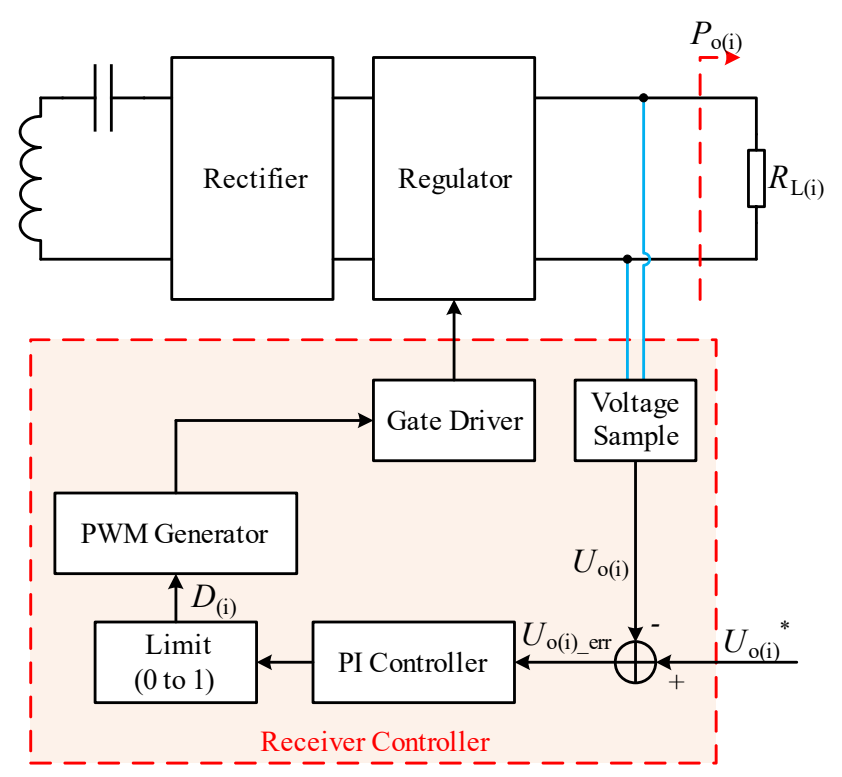

Figure 7. Scheme of receiver controller.

It can be seen that when duty cycle $D_{(i)}$ equals to 1 , the receiver has the maximum power:

$$
P_{o(i) \_ \text {max }}=\left.P_{o(i)}\right|_{D_{(i)}=1}=p_{i_{-} \text {norm }} \times \frac{\left(M_{(i)} U_{o}\right)^{2}}{R_{L(i)} L_{a}^{2}}=\cos ^{2}\left(\frac{\alpha}{2}\right) \times \frac{\left(M_{(i)} D U_{\text {rect_o }}\right)^{2}}{R_{L(i)} L_{a}^{2}}
$$

Similar to the transmitter controller, each receiver controller has two states:

State 1: $U_{(o) i}{ }^{*}<U_{o(i) \_ \text {max }}$. Figure 8a shows how the receiver controller regulates the output voltage in state 1 . If $U_{o(i)}$ is less than $U_{(o) i}^{*}$ at $t_{0}$, the error of the output voltage $U_{o(i) \_ \text {err }}$ would be positive, and, then, the PI controller would increase the duty cycle $D$ to increase $U_{o(i)}$. On the contrary, if $U_{o(i)}$ is higher than $U_{(o) i}{ }^{*}$ at $t_{0}$, the error of the output voltage $U_{o(i) \_ \text {err }}$ would be negative, so the PI controller would decrease the duty cycle $D_{(i)}$ to decrease $U_{o(i)}$. Finally, in the steady state, $U_{o(i)}$ will be equal to $U_{(o) i}{ }^{*}$ under the control of the receiver controller in state 1. 


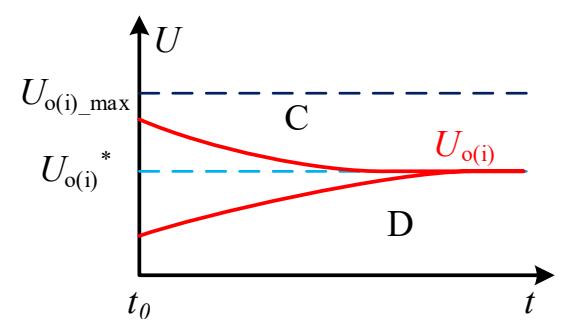

(a)

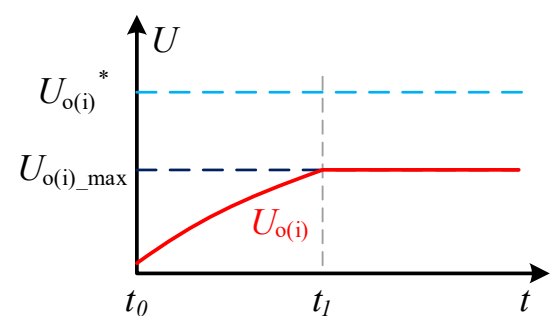

(b)

Figure 8. Working states of one receiver controller. (a) State 1 (b) State 2.

State 2: $U_{(o) i}{ }^{*} \geq U_{o(i) \_m a x}$. Figure $8 \mathrm{~b}$ shows how the receiver controller works in state 2. If $U_{o(i)}$ is less than $U_{o(i) \_m a x}$ at $t_{0}, U_{o(i) \_ \text {err }}$ will be positive, and the PI controller would try to increase $D_{(i)}$. When $D_{(i)}$ increases to 1 at $t_{1}$, the output voltage will be $U_{o(i) \_m a x}$ while the output power is $P_{o(i) \_m a x}$. $U_{o(i) \_ \text {err }}$ would still remain positive. However, $D_{(i)}$ is limited to be no greater than 1 . Thus, the output voltage will no longer increase, which means that the receiver controller is saturated. Finally, in the steady state, the output voltage will remain $U_{o(i) \_ \text {max }}$ in state 2.

In general, under the control of the receiver controller, the output voltage of the receiver in the steady state can be described as:

$$
U_{o(i)}=\min \left(U_{o(i)}^{*}, U_{o(i) \_ \text {max }}\right)=\min \left(D^{*} U_{\text {rect_o }}^{*}, U_{o(i) \_ \text {max }}\right)
$$

The controllers at the transmitter and receiver sides regulate the power in their own ways. The operation principles of the transmitter and receiver controllers are relatively simple. However, the interaction between controllers is more important.

It is shown that $P_{o(i) \_m a x}$ varies with $P_{i \_n o r m}$. However, $P_{i \_n o r m}$ is controlled by the transmitter controller. Thus, the regulation of the transmitter controller will change $P_{o(i) \_ \text {max }}$. The output power of a receiver is:

$$
P_{o(i)}=P_{o(i) \_ \text {max }} \times D_{(i)}^{2}
$$

when $P_{o(i) \_m a x}$ is changed by the transmitter controller, the receiver controller will try to maintain the output voltage, as well as the output power by adjusting $D_{(i)}$. Therefore, the transmitter controller will interfere with the receiver controllers.

Similarly, $P_{i \_ \text {max }}$ varies with $D$ in each receiver. Although $D$ is controlled by the receiver controllers, the regulation of the receiver controllers will change $P_{i \_ \text {max }}$. For the transmitter, the power absorbed from the DC power supply is:

$$
P_{i}=P_{i_{-} \max } \times p_{i_{-} \text {norm }}=P_{i_{-} \max } \times \cos ^{2}\left(\frac{\alpha}{2}\right)
$$

The transmitter will try to maintain $P_{i}$ by adjusting $P_{i \_n o r m}$ when any receiver controller changes $P_{i \_m a x}$. Therefore, the receiver controllers will also interfere with the transmitter controller.

Both the transmitter and receiver controllers will participate in the regulation process before the system enters the steady state, with the two types of controllers affecting each other during the regulation. The power distribution is the result of the interaction between the controllers.

The following relationship is derived by combining (16) and (17):

$$
\sum_{i=1}^{n} P_{o(i)}=\eta P_{i}
$$


From (20), the ratio of $P_{o_{-} \max }$ between the receivers can be calculated as:

$$
P_{o(i) \_ \text {max }}: P_{o(j) \_ \text {max }}=p_{i \_n o r m} \times \frac{\left(M_{(i)} U_{o}\right)^{2}}{R_{L(i)} L_{a}^{2}}: p_{i \_n o r m} \times \frac{\left(M_{(j)} U_{o}\right)^{2}}{R_{L(j)} L_{a}^{2}}=\frac{M_{(i)}^{2}}{R_{L(i)}}: \frac{M_{(j)}^{2}}{R_{L(j)}}
$$

where $i$ and $j$ are the serial numbers of receivers.

Under the assumption that the efficiency is constant and by differentiating (28) and (29) with respect to $P_{i}$, the following equations can be derived:

$$
\left\{\begin{array}{l}
\sum_{i=1}^{n} \frac{\mathrm{d} P_{o(i)}}{\mathrm{d} P_{i}}=\eta \\
\frac{\mathrm{d} P_{o(i)} \max }{\mathrm{d} P_{i}}: \frac{\mathrm{d} P_{o(j) \_m a x}}{\mathrm{~d} P_{i}}=\frac{M_{(i)}^{2}}{R_{L(i)}}: \frac{M_{(j)}^{2}}{R_{L(j)}}
\end{array}\right.
$$

Figure 9 shows an example illustrating the power distribution between the receivers. In this example, a multi-receiver WPT system with three receivers is presented. The power distribution of the entire system in the steady state as $P_{i}^{*}$, which varies from low to high. The blue lines indicate the variations in $P_{i}$ and $P_{o_{-} \text {sum }}$ with $P_{i}^{*}$. The gray area between $P_{i}$ and $P_{o_{-} \text {sum }}$ represents the power loss $P_{\text {loss }}$. The other lines indicate the variation in the output power of the receivers with $P_{i}^{*}$.

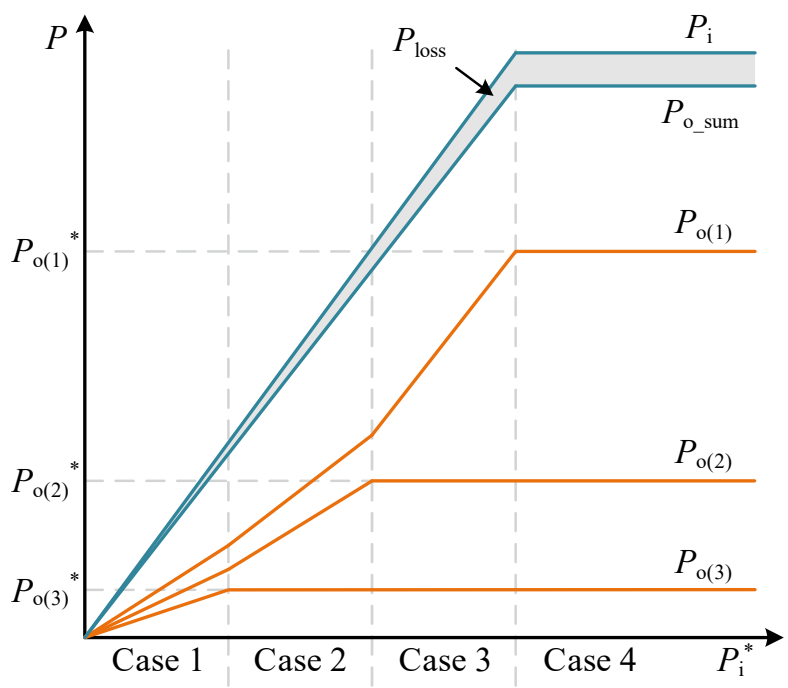

Figure 9. Example of power distribution in the steady state.

\subsection{Four Typical Case Study in the Proposed Structure}

Four typical stages in this example are analyzed in detail, and each specific case is represented by in Figure 10.

Case 1: $P_{i}^{*}$ is low enough, and the transmitter controller will set $P_{i \_n o r m}$ to a low level to ensure that $P_{i}$ is equal to $P_{i}^{*}$. Consequently, $P_{o_{-} \max }$ of each receiver will be lower than $P_{o}^{*}$, and all the receiver controllers will work in state $2 . P_{o}$ of every receiver is equal to $P_{0 \_} \max$ under the control of the receiver controller. The above analysis shows that $P_{i}=P_{i}^{*}$ and $P_{o}=P_{O_{-} \max }$. Substituting the above relationships into (30), the slope of $P_{o}$ can be derived based on (22) as:

$$
\text { Slope }=\frac{P_{o(i)}}{P_{i}^{*}}=\eta \frac{P_{o(i)}}{\sum_{i=1}^{3} P_{o(i)}}=\eta \frac{\frac{U_{i}^{2}}{L_{a}^{2}} \times \frac{\left(M_{(i)} D_{(i)}\right)^{2}}{R_{L(i)}}}{\frac{U_{i}^{2}}{L_{a}^{2}} \times \sum_{i=1}^{3} \frac{\left(M_{(i)} D_{(i)}\right)^{2}}{R_{L(i)}}}=\eta \frac{\frac{M_{(i)}^{2}}{R_{L(i)}}}{\frac{M_{(1)}^{2}}{R_{L(1)}}+\frac{M_{(2)}^{2}}{R_{L(2)}}+\frac{M_{(3)}^{2}}{R_{L(3)}}}, i=1,2,3
$$




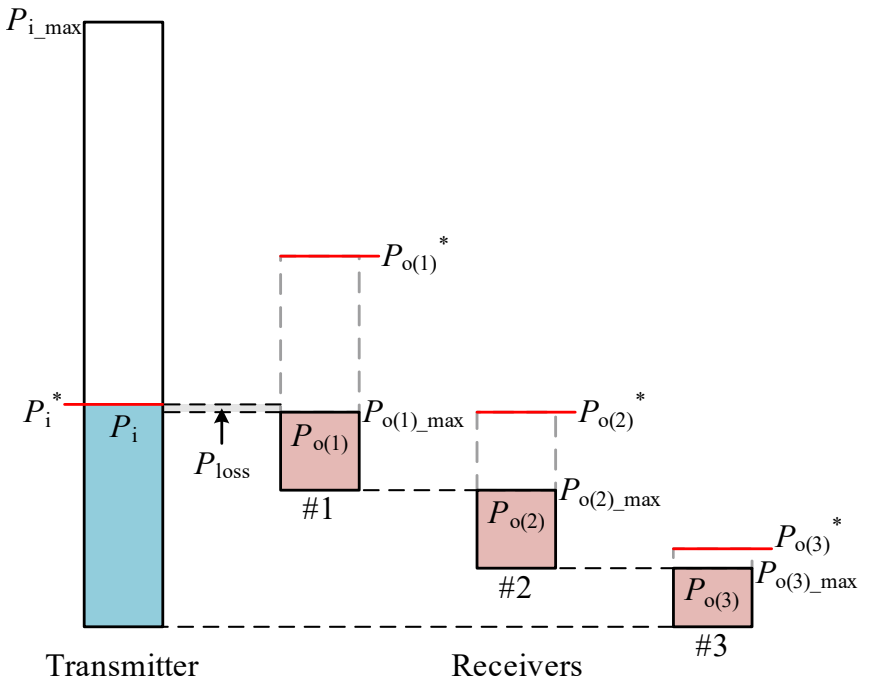

(a)

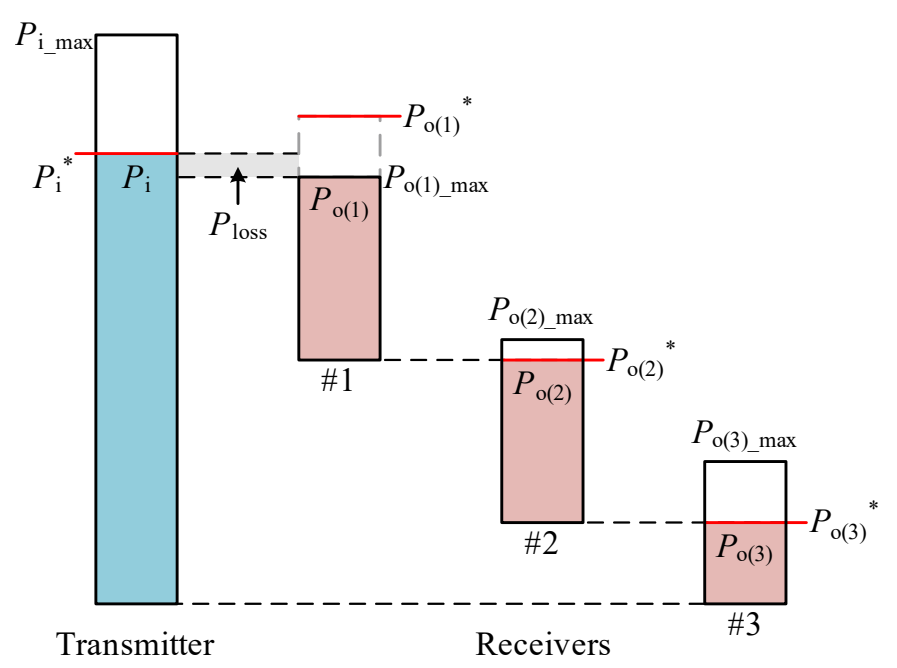

(c)

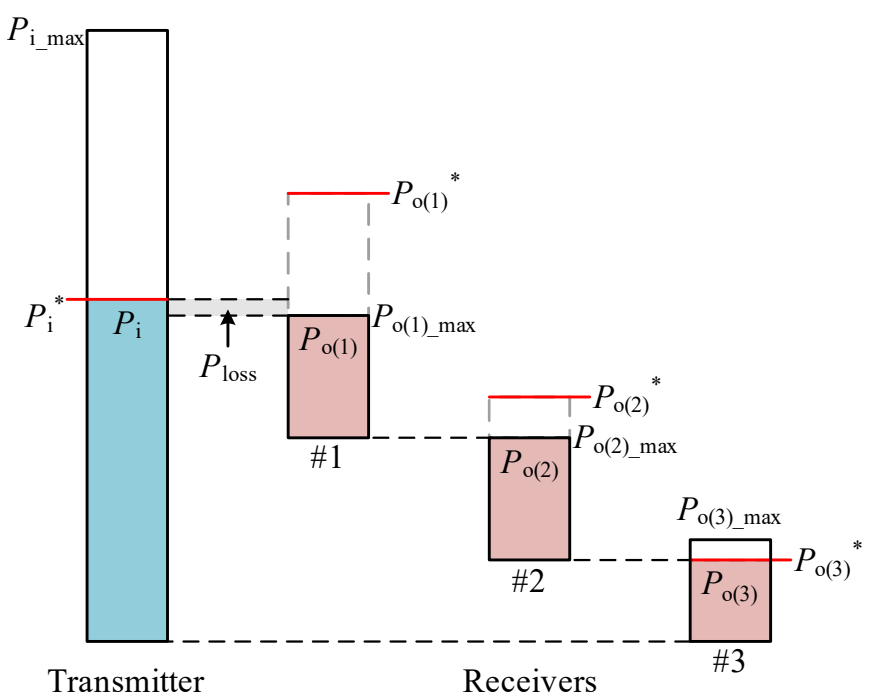

(b)

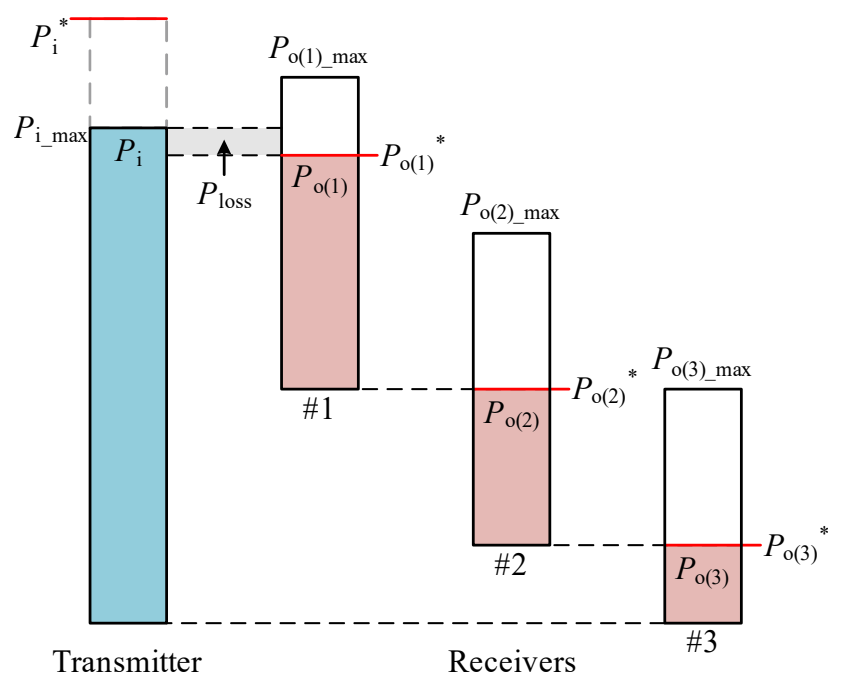

(d)

Figure 10. Four power distribution cases (a) Case 1, (b) Case 2, (c) Case 3, (d) Case 4.

Case 2: When the system already works in case 1 and then increases $P_{i}^{*}$ until one of the receiver controllers transfers to state 1 , the system would transfer to case 2 . The transmitter controller will increase $P_{i \_n o r m}$ to increase $P_{i}$, thereby increasing $P_{o_{-} \text {max }}$ of every receiver. For the receiver \#3, $P_{o(3) \_ \text {max }}$ will increase beyond $P_{o(3)}{ }^{*}$. Therefore, the controller of receiver \#3 would enter state 1 and $P_{o(3)}$ is equal to $P_{o(3) \_m a x}$. The receiver controllers of receivers \#1 and \#2 remain in state 2. To sum up, in case $2, P_{i}=P_{i}^{*}, P_{o(1)}=P_{o(1) \_m a x}$, $P_{o(2)}=P_{o(2) \_ \text {max }}$, and $P_{o(3)}=P_{o(3)}{ }^{*}$. Substituting the above relationships into (31), the slope of $P_{o}$ in case 2 can be derived as:

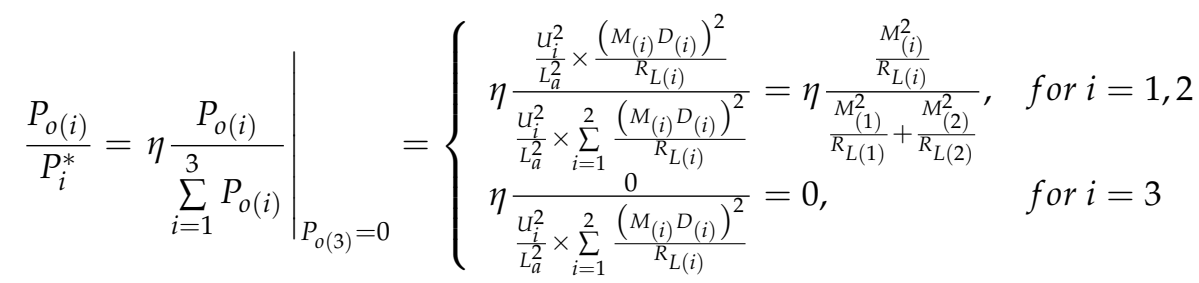

Case 3: When the system already works in case 2 and then further increases $P_{i}^{*}$ until another receiver controller transfers to state 1 , the system would transfer to case 3 . The transmitter controller will further increase $P_{i \_n o r m}$ to increase $P_{i}$, thereby increasing $P_{0 \_} \max$ 
of every receiver. In this example, the controller of receiver \#2 will transfer to state 1. Meanwhile, the receiver controller of receiver $\# 1$ remains in state 2 and receiver controller of receiver $\# 3$ remains in state 1 . To sum up, in case $3, P_{i}=P_{i}^{*}, P_{o(1)}=P_{o(1) \_\max }, P_{o(2)}=P_{o(2)}{ }^{*}$, and $P_{o(3)}=P_{o(3)}$. Substituting the above relationships into (30), the slope of $P_{o}$ in case 3 can be derived as:

$$
\frac{P_{o(i)}}{P_{i}^{*}}=\left.\eta \frac{P_{o(i)}}{\sum_{i=1}^{3} P_{o(i)}}\right|_{P_{o(2)}=P_{o(3)}=0}=\left\{\begin{array}{l}
\eta \frac{\frac{u_{i}^{2}}{L_{a}^{2}} \times \frac{\left(M_{(1)} D_{(1)}\right)^{2}}{R_{L(1)}}}{\eta \frac{u_{i}^{2}}{L_{a}^{2}} \times \frac{\left(M_{(1)} D_{(1)}\right)^{2}}{R_{L(1)}}}=\eta, \quad i=1 \\
\eta \frac{u_{i}^{2}}{L_{a}^{2}} \times \frac{\left(M_{(1)} D_{(1)}\right)^{2}}{R_{L(1)}}=0, \quad i=2,3
\end{array}\right.
$$

Case 4: When $P_{i}^{*}$ is set high enough, the system will enter case 4 . In this case, $P_{i \_n o r m}$ will be saturated at 1 under the control of the transmitter controller, which means that the transmitter works in state 2 . Because $P_{i_{-} \text {norm }}$ maintains its maximum value of 1 , the $P_{o_{-}} \max$ values of each receiver reach their maximum values and are higher than $P_{o}^{*}$. Consequently, all the receiver controllers will work in case 1 . To sum up, in this case, $P_{i}=P_{i_{-} \max }$ and $P_{o}=P_{o}^{*}$. Substituting the above relationships into (26), the slope of $P_{o}$ in case 4 can be derived as:

$$
\frac{P_{o(i)}}{P_{i}^{*}}=\left.\frac{P_{o(i)}}{P_{i}^{*}}\right|_{P_{o(1)}=P_{o(2)}=P_{o(3)}=0}=\frac{0}{P_{i}^{*}}=0, i=1,2,3
$$

With the slope information of each case, the power distribution in this system in the steady state can be calculated.

This example has three receiver and four cases, and the number of cases $n_{\text {case }}$ is determined by the number of receivers:

$$
n_{\text {case }}=n+1
$$

The total charging power is non-cooperative game, the Karush-Kuhn-Tucker could be applied to optimize the gaming relation among the receivers. The power can be inserted into the Lagrangian function as

$$
L=-\sum_{i=1}^{n} w_{i} \ln \left(p_{i}+1\right)+v\left(\sum_{i=1}^{n} p_{i}-p_{\text {total }}\right)
$$

Then the partial derivatives can be derived as

$$
\begin{gathered}
\frac{\partial L}{\partial p_{i}}=-\frac{w_{i}}{p_{i}+1}+v=0, \text { for } i=1, \ldots, n \\
\frac{\partial L}{\partial v}=\sum_{i=1}^{n} p_{i}-p_{\text {total }}=0
\end{gathered}
$$

The KKT candidate point can be solved as

$$
\begin{gathered}
p_{i}=\frac{w_{i}\left(p_{\text {total }}+n\right)-\sum_{i=1}^{n} w_{i}}{\sum_{i=1}^{n} w_{i}}, \text { for } v=\frac{w_{i}}{p_{i}+1} \\
p_{i}=P_{i}^{*}, \text { for } v=0
\end{gathered}
$$

Since the Lagrangian function is convex, the Hessian of the Lagrangian function is always positive definite and the derivative is the global optimal point. Then, the calculated power divisions are achieved through the duty cycle control of the DC-DC converters in the receivers. The proposed algorithm is linear, and is proportional to $\mathrm{O}(n)$. 
For a given receiver, the combined Langrangian function can be calculated as

$$
L_{i}=u_{i}+\lambda_{i}\left(\sum_{i=1}^{n} p_{i}-p_{\text {total }}\right)
$$

where $\lambda_{i}$ is the Lagrange multiplier. Under the Karush-Kuhn-Tucker conditions, the optimization problem can be given as

$$
\frac{\partial L_{i}}{\partial P_{i}}=-\frac{w_{i}}{p_{i}+1}+\lambda_{i}=0
$$

Then, the charging power of each receiver is competing under the balanced condition at the power of $p_{\text {total }}$, therefore,

$$
p_{i}=\frac{w_{i}\left(p_{\text {total }}+n\right)}{\sum_{i=1}^{n} w_{i}}-1
$$

Then, the distributed power is unique. In our application, the implementation of competitive mechanism is realized by searching $\lambda_{i}$ when the receivers need to compete on the limited total available transmitting power $p_{\text {total }}$, as shown in the flow chart in Figure 11.

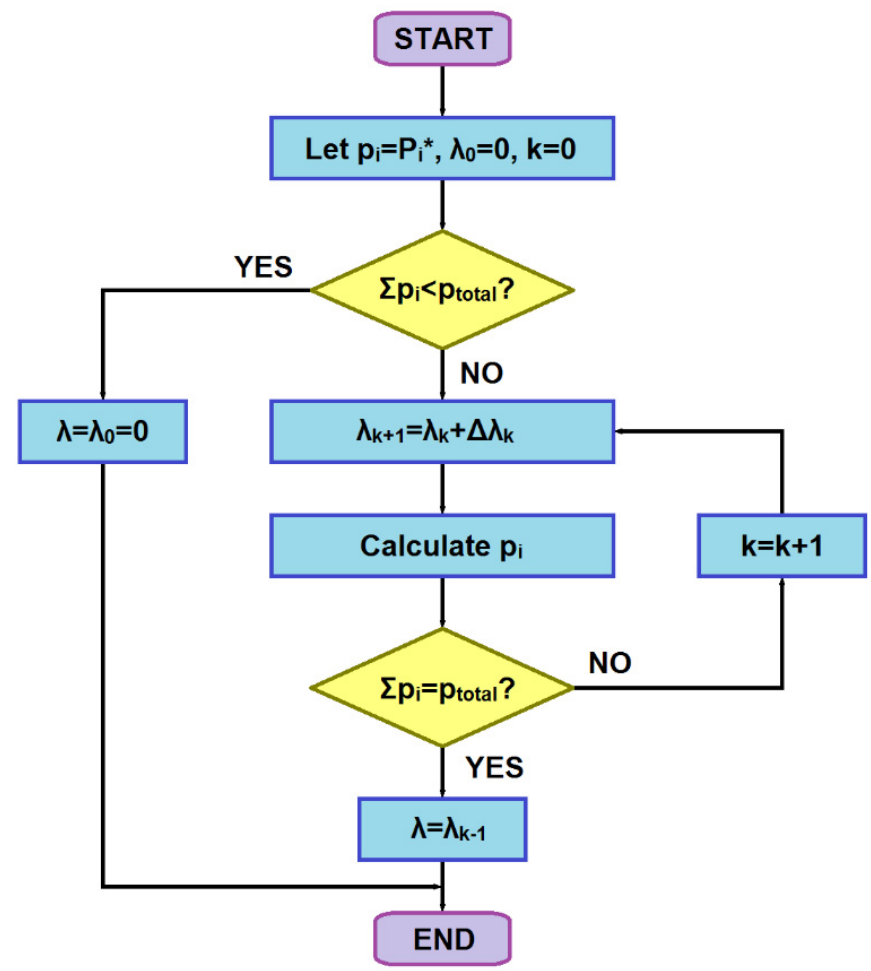

Figure 11. Flow chart of the competition algorithm.

The power distribution is the result of the interplay among the receivers. The total power is limited by the transmitter, and all the receivers compete for more power until their demands are met. Receivers with higher mutual inductance and low load resistance have an advantage. Finally, there will be an equilibrium. The result is that some receivers get sufficient power, while some do not. The balance itself is the result of power distribution. With the increase in the transmitter power capacity, more and more receivers can obtain the required power, and they do not compete for more power. They maintain their existing power, while others still compete for more power. 
In addition, the power distribution depends on the mutual positions of elements too. So, constant, defined, and identical position between elements should be expected. Figure 12 gives the magnetic field distribution of the coils. Since the receiving coils are aligned diagonally, magnetic induction lines from one receiving coil hardly pass through the other two receiving coils. Therefore, the cross-coupling among the receiving coils can be neglected.

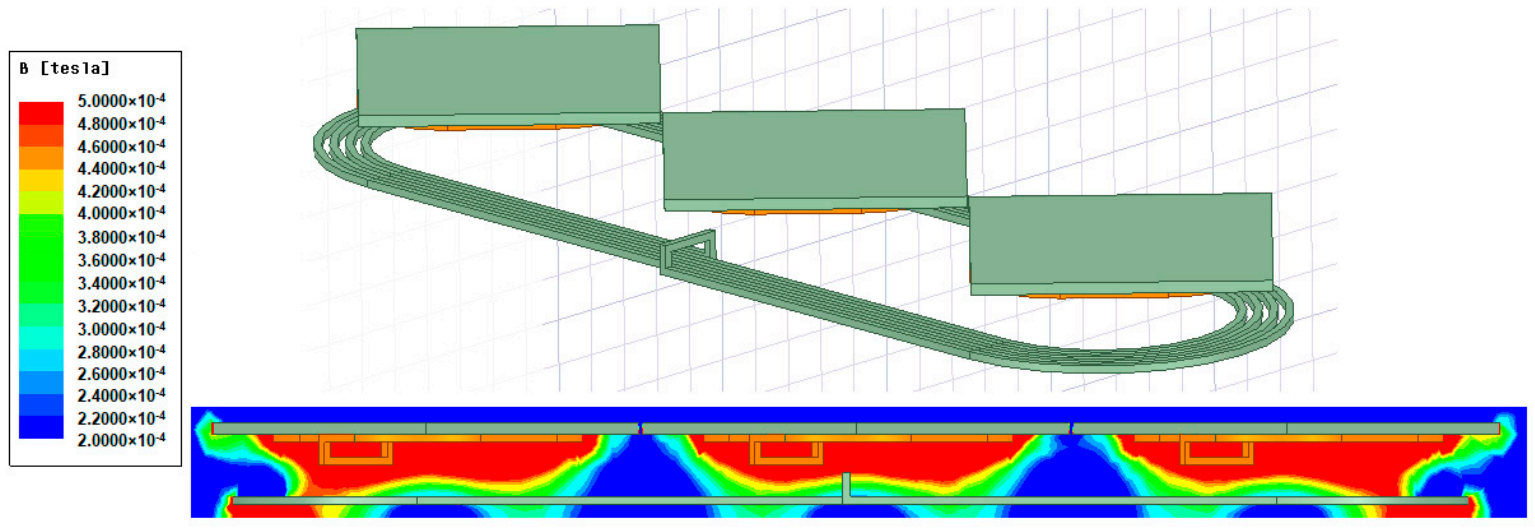

Figure 12. Magnetic field distribution of the coils.

\section{Results}

An experimental multi-receiver WPT system has been built to verify the validity of the theoretical analysis.

The experimental setup is shown in Figure 13. It has one transmitter and three individual receivers. The model of all semiconductor switches in the system is Infineon IHW50N65R5, and the model of receiver rectifier diodes is CREE D3065C5.

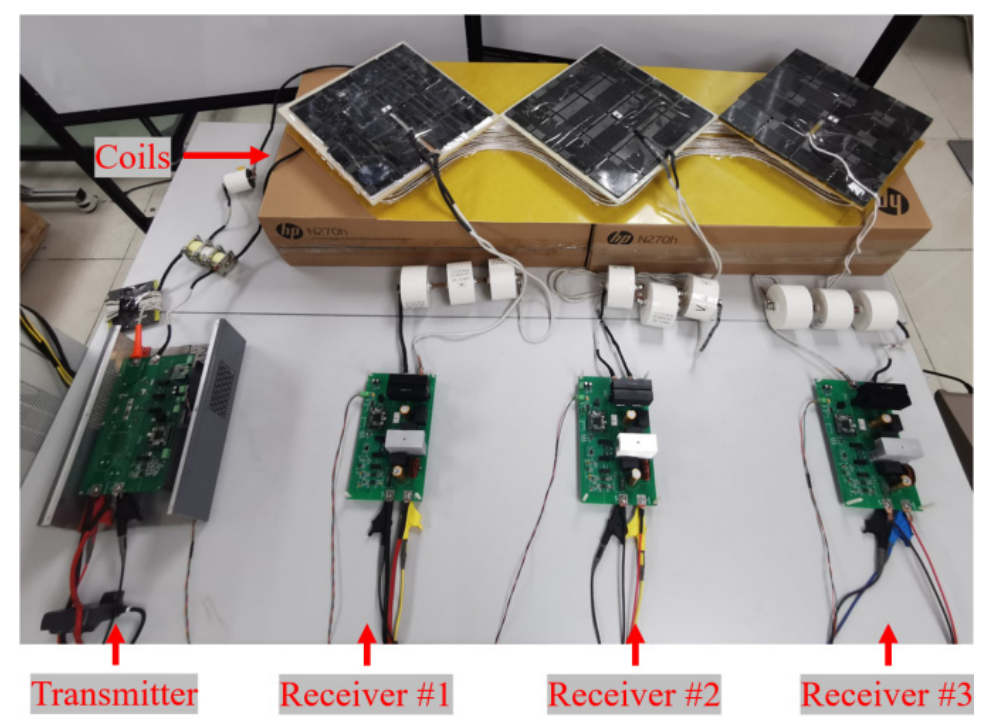

Figure 13. Photograph of the experimental setup.

The coil structure is shown in Figure 14. The transmitting coil has three slots designed for three receivers. Its winding consists of eight turns of Litz wire. All the receiving coils have the same structure and size. They are square-shaped with ferrite bricks over them to augment the mutual inductance. The gap between the transmitting and receiving coils is $5 \mathrm{~cm}$. 


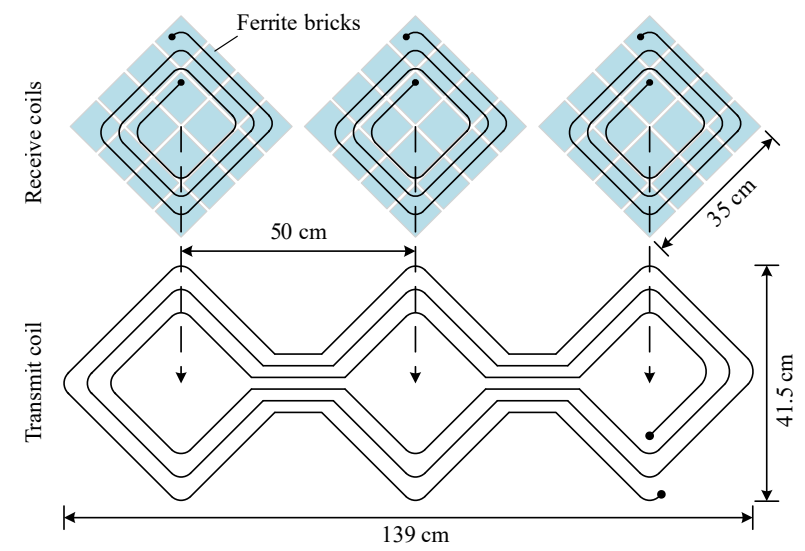

(a)

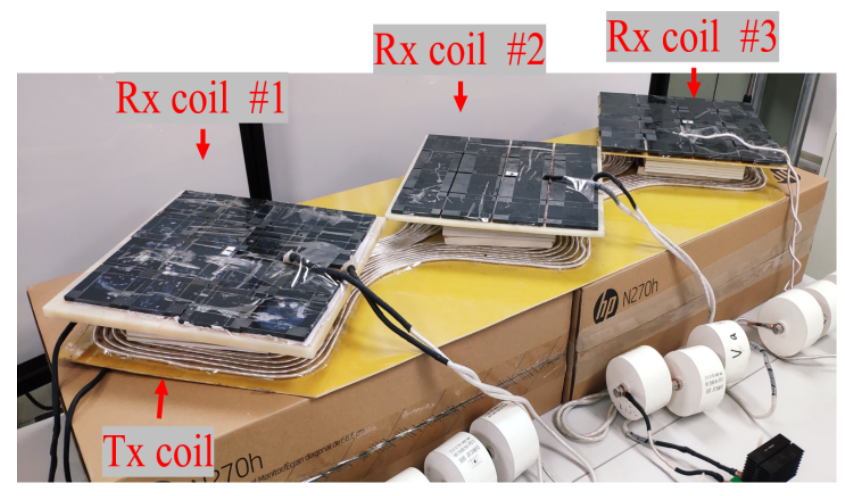

(b)

Figure 14. Coil structure. (a) Diagram. (b) Photograph.

The configuration of this experimental setup is listed in Table $1 . \omega L_{a}$ of the LCC compensation circuit at the transmitting side is designed to be $10 \mathrm{ohms}$. The designated parameters of the compensation circuit are derived from the analysis.

Table 1. Parameters of the experimental setup.

\begin{tabular}{cccc}
\hline & Symbol & Parameter & Value \\
\hline & $f$ & Frequency & $85 \mathrm{kHz}$ \\
& $U_{i}$ & DC power supply voltage & $110 \mathrm{~V}$ \\
\hline \multirow{3}{*}{ Transmitter } & $L_{1}$ & Tx coil self-inductance & $91.53 \mu \mathrm{H}$ \\
& $C_{1 b}$ & Tx compensation capacitor & $187.24 \mathrm{nF}$ \\
& $C_{1 a}$ & Tx compensation capacitor & $47.97 \mathrm{nF}$ \\
& $L_{a}$ & Tx compensation inductor & $18.85 \mu \mathrm{H}$ \\
\hline \multirow{3}{*}{ Receiver \#1 } & $L_{2(1)}$ & Rx coil self-inductance & $42.89 \mu \mathrm{H}$ \\
& $M_{(1)}$ & Mutual inductance to Tx coil & $16.30 \mu \mathrm{H}$ \\
& $C_{2(1)}$ & Compensation capacitor & $81.69 \mathrm{nF}$ \\
\hline \multirow{2}{*}{ Receiver \#2 } & $L_{2(2)}$ & Rx coil self-inductance & $42.20 \mu \mathrm{H}$ \\
& $M_{(2)}$ & Mutual inductance to Tx coil & $14.51 \mu \mathrm{H}$ \\
& $C_{2(2)}$ & Compensation capacitor & $85.15 \mathrm{nF}$ \\
\hline \multirow{2}{*}{ Receiver \#3 } & $L_{2(3)}$ & Rx coil self-inductance & $52.14 \mu \mathrm{H}$ \\
& $M_{(3)}$ & Mutual inductance to Tx coil & $17.61 \mu \mathrm{H}$ \\
& $C_{2(3)}$ & Compensation capacitor & $67.12 \mathrm{nF}$ \\
\hline
\end{tabular}

The switching frequency of the receiver regulator is $50 \mathrm{kHz}$. The control algorithm is realized by programming using DSP controllers, with every transmitter and receiver having an individual DSP controller. The loads of the receivers were electric loads in the constant resistance (CR) mode. A power analyzer is connected to record the powers. In the experiment, the gate driver UCC21520 and PI controller dsp28335 have been used. The current and voltage signals are sampled and differentially amplified by AMC1302. The signal is collected through AD7682 and then transmitted back to the DSP.

The static experiment was conducted to verify the validity of the theoretical analysis, where the input power was set at the maximum value. The parameters of the controllers and the loads are shown in Table 2 . In this experiment, all the receivers were active. $P_{i}^{*}$ was swept from low to high. Each time $P_{i}^{*}$ changed, the controllers would regulate the power and, thus, the system would enter steady state again. The three receivers are terminated with $12 \Omega, 14 \Omega$, and $20 \Omega$ loads, respectively. 
Table 2. Parameters of controllers and loads.

\begin{tabular}{ccc}
\hline & Symbol & Value \\
\hline \multirow{2}{*}{ Load Resistance } & $R_{L(1)}$ & $12 \Omega$ \\
& $R_{L(2)}$ & $14 \Omega$ \\
& $R_{L(3)}$ & $20 \Omega$ \\
\hline \multirow{2}{*}{ Instruction output voltage } & $\left.U_{o(1)}\right)^{*}$ & $80 \mathrm{~V}$ \\
& $U_{o(2)^{*}}$ & $70 \mathrm{~V}$ \\
Equivalent instruction output power & $U_{o(3)^{*}}$ & $60 \mathrm{~V}$ \\
& $P_{o(1)^{*}}$ & $533.3 \mathrm{~W}$ \\
& $P_{o(2)^{*}}$ & $350 \mathrm{~W}$ \\
& $P(3)^{*}$ & $120 \mathrm{~W}$ \\
\hline
\end{tabular}

The result of the static experiment is shown in Figure 15. The optimal efficiency in the simulation is equal to $88 \%$. With the increase in $P_{i}^{*}, P_{i}$ is always lower than $P_{i}^{*}$, indicating that $P_{i}$ is limited under the regulation of the transmitter controller. The total output power $P_{o_{-} \text {sum }}$ increases with the increase in $P_{i}{ }^{*}$. The difference between $P_{i}$ and $P_{o_{-} \text {sum }}$ is the power loss of the entire system $P_{\text {loss }}$. The power distribution of this experiment is comprised of four cases: $P_{o}$ curves have different slopes in different cases. With the increase in $P_{i}^{*}$, all the receivers absorb more power until the output power $P_{0}$ reaches its equivalent instructed output power $P_{o}{ }^{*}$. Finally, all the receivers reach their $P_{o}^{*}$ as $P_{i}^{*}$ is high enough.

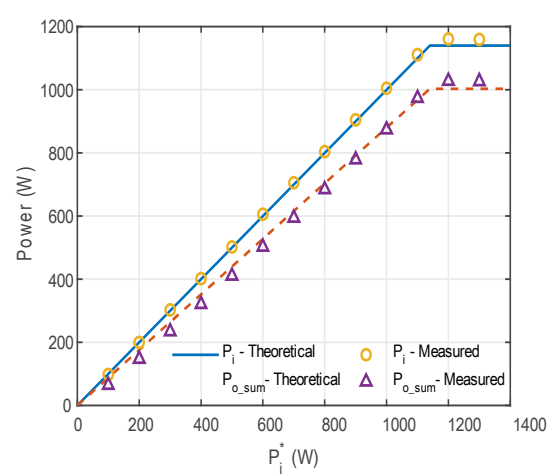

(a)

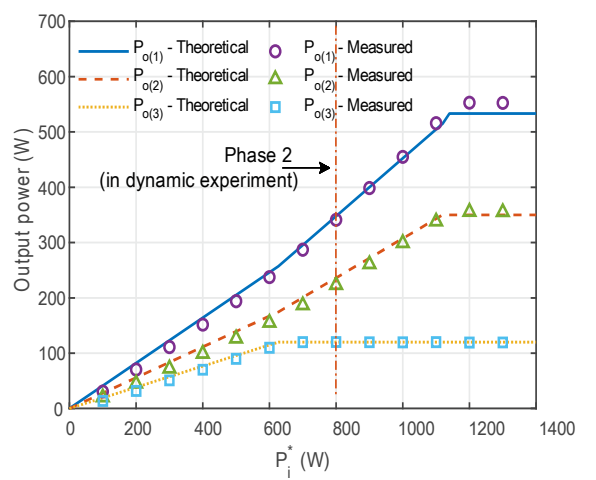

(b)

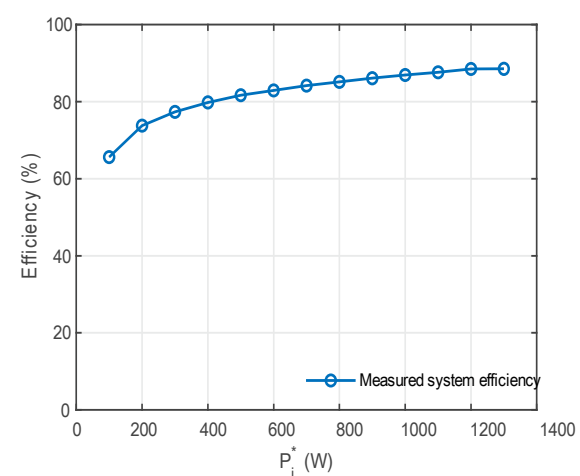

(c)

Figure 15. Power distribution in each case, (a) Case 1, (b) Case 2, (c) Case 3.

The comparison shows that the experimental result is consistent with the theoretical result. However, there are some errors between the measured and theoretical values. The errors originate from the following sources: (1) Theoretical result is obtained under the assumption that the efficiency is constant; in the practical system, the efficiency increases with the increase in the power. (2) Voltage drops of the wire and semiconductor devices are not considered in the theoretical analysis. (3) The practical components of the compensation circuits and coil have tolerances. Thus, the system deviates from the resonance state. (4) The harmonic components are ignored in the theoretical analysis. Nonetheless, the theoretical analysis is in good agreement with the experimental result with acceptable errors.

A dynamic experiment is performed to verify the flexibility of the proposed method, where the input power is increased dynamically from 0 to the maximum value. In this experiment, the working condition was set to simulate a practical working condition. In a practical working condition, the system parameters may vary dynamically. For example, the coil position, load resistance, and number of receivers may dynamically change while the system is running. The proposed control method is expected to handle these variations.

This experiment simulated the condition wherein the number of receiver changes from 2 to 3 . The setup is the same as the setup in the static experiment. This procedure 
consists of two phases. In phase 1, only receivers \#1 and \#3 are operated in the WPT system while receiver \#2 is disabled. In phase 2, all the receivers are operated. First, the system works in phase 1 and is in steady state, then receiver \#2 joins the WPT system and the system turns to phase 2 . With the joining of receiver \#2, the controllers regulate the power among the receivers. Finally, after the transient regulation of the controllers, the system would enter the steady state of phase 2 . A power analyzer is employed to record the input and output power continuously with a period of $10 \mathrm{~ms}$.

Under the theoretical power distribution condition in the steady state of phase 1, the optimal efficiency is $88 \%$. As the condition of phase 2 is the same as that in the static experiment, the power distribution state is steady. The theoretical power distribution in both phases 1 and 2 can be determined. Figure 16 shows the power variation when the system moves from phase 1 to phase 2 . At the beginning, the system is in the steady state of phase 1 . The output power of receiver \#2 was 0 as it is disabled, and the power only flows to receiver \#3. As soon as the receiver \#2 is activated at $43.2 \mathrm{~s}$, the controller starts to increase the output power of receiver \#2. The output power of receiver \#3 decreases to the steady value in phase 2 . The output power of receiver \#3 decreases first, then increases, and finally maintains the same value. The regulation process lasts for approximately $3 \mathrm{~s}$. Finally, the system returns to the steady state. In Table 3, the theoretical and measured power distributions in the steady state are compared. The measured power values match well with the theoretical power values with the maximum error of $-5.50 \%$.

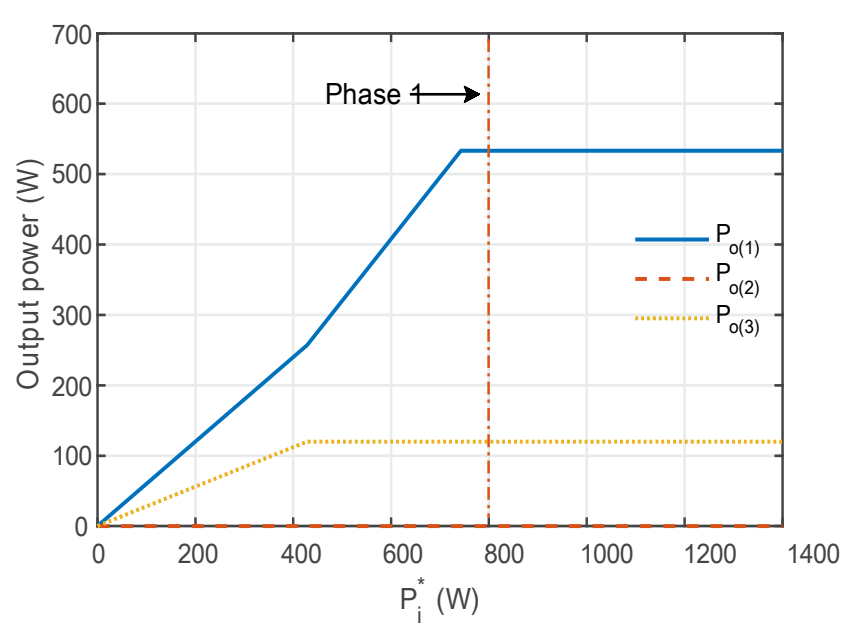

(a)

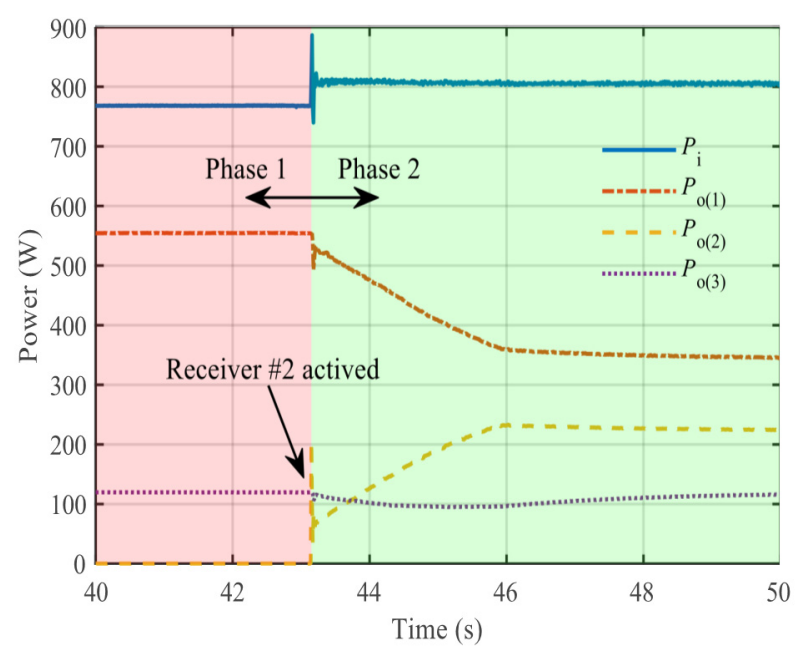

(b)

Figure 16. Dynamic experiment result. (a) Theoretical power distribution in phase 1. (b) Power variation from phase 1 to phase 2.

Table 3. Power in the steady state in the dynamic experiment.

\begin{tabular}{cccccc}
\hline & & $\boldsymbol{P}_{\boldsymbol{i}}$ & $\boldsymbol{P}_{\boldsymbol{o}(\mathbf{1})}$ & $\boldsymbol{P}_{\boldsymbol{o}(\mathbf{2})}$ & $\boldsymbol{P}_{\boldsymbol{o}(\mathbf{3})}$ \\
\hline \multirow{4}{*}{ Phase 1 } & Theoretical & $768.6 \mathrm{~W}$ & $533.3 \mathrm{~W}$ & $0.0 \mathrm{~W}$ & $120.0 \mathrm{~W}$ \\
& Measured & $768.5 \mathrm{~W}$ & $554.7 \mathrm{~W}$ & $0.0 \mathrm{~W}$ & $119.7 \mathrm{~W}$ \\
& Error & $-0.01 \%$ & $4.01 \%$ & - & $-0.25 \%$ \\
\hline \multirow{3}{*}{ Phase 2 } & Theoretical & $800.0 \mathrm{~W}$ & $348.8 \mathrm{~W}$ & $236.2 \mathrm{~W}$ & $120.0 \mathrm{~W}$ \\
& Measured & $805.7 \mathrm{~W}$ & $343.8 \mathrm{~W}$ & $223.2 \mathrm{~W}$ & $119.6 \mathrm{~W}$ \\
& Error & $0.71 \%$ & $-1.43 \%$ & $-5.50 \%$ & $-0.33 \%$ \\
\hline
\end{tabular}

The dynamic experimental result demonstrates that the proposed control method can handle the dynamic variation in the number of receivers. The power is redistributed under the combined action of all the controllers following a change in the number of receivers in the system. The control method demonstrates flexibility and robustness in the dynamic 
experiment. The controller works as expected even when some of the parameters vary while the system is in operation.

\section{Discussion}

In a practical scenario, $P_{i}^{*}$ of the transmitter controller should be the same or only slightly lower than the rated power of the DC power supply of the transmitter to prevent it from overloading. The $P_{i}^{*}$ limits the maximum power of the entire system. $P_{o}^{*}$ of the receivers should be the required power of their loads. However, the practical output power $P_{o}$ may be lower than the required power due to the limit of the transmitter power capacity. The experimental setup adopts a transmitting coil designed especially for three receivers. However, the transmitting coil design is not limited. Coils with any shape, size, number of receivers, etc., are acceptable for the proposed control method. Receivers with higher power demand should be equipped with bigger receiving coil to achieve higher mutual inductance. Otherwise, the receiver may face disadvantage in the competition.

The proposed method distributes power unequally, but the competition mechanism makes dynamic distribution possible. Receivers can join or quit the system freely, and the receiver power demand can also vary dynamically. No matter how the system varies, the system will redistribute the power automatically. This control method can handle massive receivers without reprogramming, and will not become complicated with the increase in the number of receivers. Therefore, the system can expand easily.

Table 4 gives the comparison between the proposed method and some representative existing methods, and the advantages and disadvantages are listed. It demonstrates the superiority of the proposed method over previous methods. In the comparison, algorithm complexity is judged by the time and space consumed at a given input of size $\mathrm{n}$ [27] The controller algorithms lower than $\mathrm{O}(\mathrm{n} \log n)$, such as $\mathrm{O}(\operatorname{logn})$ and $\mathrm{O}(\mathrm{n})$, are defined as "simple" whereas those higher than $\mathrm{O}(\mathrm{n} \log n)$, such as $\mathrm{O}\left(\mathrm{n}^{\wedge} \mathrm{k}\right)$ and $\mathrm{O}\left(\mathrm{k}^{\wedge} \mathrm{n}\right)$, are defined as "complicated". Circuit configuration complexity is based on equivalent circuit topology. If the topology structure satisfies Karp-Lipton condition [28], it is defined as "simple", otherwise it is defined as "complicated". Dynamic distribution is judged by whether the circuit can distribute power flexibly with the change of outside factors, such as load, receiver number, or charging distance. Scalability is judged by whether this system can be applied in other multi-receiver scenarios with only changing the parameters instead of system structure. This is mainly determined by circuit topology, operating frequency and dynamic distribution.

Table 4. Comparison between existing methods.

\begin{tabular}{cccccc}
\hline Method & $\begin{array}{c}\text { Controller } \\
\text { Algorithm }\end{array}$ & $\begin{array}{c}\text { Main Circuit } \\
\text { Configuration }\end{array}$ & $\begin{array}{c}\text { Dynamic } \\
\text { Distribution }\end{array}$ & Scalability & Additional Requirement \\
\hline Impedance matching [4,15,20] & None & Simple & No & Low & - \\
Multi-frequency method [19] & None & Complicated & No & Low & Multiple inverters \\
Time-sharing method [18] & Simple & Complicated & Yes & Low & Capacitor switching array \\
Frequency communication & Simple & Simple & Yes & High & High accuracy frequency \\
method [16] & Complicated & Simple & Yes & High & - \\
Game-theory-based control [21] & Simple & Simple & Yes & High & - \\
Proposed method & & & &
\end{tabular}

The proposed method has a constant-voltage output characteristic. In the future, the configuration can be set to constant current output by modifying the compensation circuit and receiver controller to suit applications, such as charging batteries or supercapacitors.

\section{Conclusions}

A power distribution control method for a multi-receiver WPT system is proposed in this paper. By employing double-side control, the power from transmitter distributes to multiple receivers automatically. Receivers acquire power by competition, and the 
receivers with higher mutual inductance is easier to gain higher power. This procedure is dynamic, so the power will be redistributed when the system parameters varied. Hence, this method can handle various occasions such as the number of receivers changing, receiver power demands changing or coil position (mutual inductance) changing. For receivers, the received power will not exceed their demands, while the transmitter will not be overloaded at any given time. The control algorithm and main circuit configuration are simple, and the communication channel is not required. The increase in the number of receivers will not increase the complexity. Thus, the number of receivers can be increased easily. Static experiment with fixed system parameters indicates the power distribution result can be accurately predicted based on the circuit and controller parameters. Dynamic experiment with dynamically varying system parameters demonstrates the reliability, scalability, flexibility, and generality of this method. The proposed WPT system is compared with several others. The classification is derived based on algorithm theory and circuit topology. The characteristics are determined through mathematical calculations based on the results provided in those references.

Author Contributions: Conceptualization, Y.Z. and X.C.; methodology, Y.Z.; validation, Y.Z., H.Z.; investigation, R.Z.; writing—original draft preparation, H.Z.; writing—review and editing, Z.W.; supervision, Y.Z.; funding acquisition, Y.Z. All authors have read and agreed to the published version of the manuscript.

Funding: This research was funded by Doctoral Foundation of Southwest University of Science and Technology, grant number 20zx7121 \& 19zx7156.

Acknowledgments: We would like to thank the reviewers for their valuable review comments. Some of the revisions have been taken directly from the reviewers' suggestions.

Conflicts of Interest: The authors declare no conflict of interest.

\section{References}

1. Cannon, L.; Hoburg, J.F.; Stancil, D.D.; Goldstein, S.C. Magnetic Resonant Coupling As a Potential Means for Wireless Power Transfer to Multiple Small Receivers. IEEE Trans. Power Electron. 2009, 24, 1819-1825. [CrossRef]

2. Kurs, A.; Moffatt, R.; Soljačić, M. Simultaneous mid-range power transfer to multiple devices. Appl. Phys. Lett. $2010,96,044102$. [CrossRef]

3. Ahn, D.; Hong, S. Effect of Coupling between Multiple Transmitters or Multiple Receivers on Wireless Power Transfer. IEEE Trans. Ind. Electron. 2013, 60, 2602-2613. [CrossRef]

4. Fu, M.; Zhang, T.; Ma, C.; Zhu, X. Efficiency and Optimal Loads Analysis for Multiple-Receiver Wireless Power Transfer Systems. IEEE Trans. Microw. Theory Tech. 2015, 63, 801-812. [CrossRef]

5. Hu, X.; Wang, Y.; Jiang, Y.; Lei, W.; Dong, X. Maximum Efficiency Tracking for Dynamic Wireless Power Transfer System Using LCC Compensation Topology. In Proceedings of the 2018 IEEE Energy Conversion Congress and Exposition (ECCE 2018), Portland, OR, USA, 23-27 September 2018; pp. 1992-1996.

6. Liu, W.; Chau, K.T.; Lee, C.H.T.; Jiang, C.; Han, W.; Lam, W.H. Multi-Frequency Multi-Power One-to-Many Wireless Power Transfer System. IEEE Trans. Magn. 2019, 55, 1-9. [CrossRef]

7. Zhou, J.; Gao, Y.; Huang, X.; Hu, S.; Fang, Y. Voltage transfer ratio analysis for multireceiver resonant power transfer systems. IET Power Electron. 2016, 9, 2795-2802. [CrossRef]

8. Kuang, J.; Luo, B.; Zhang, Y.; Hu, Y.; Wu, Y. Load-Isolation Wireless Power Transfer With K-Inverter for Multiple-Receiver Applications. IEEE Access 2018, 6, 31996-32004. [CrossRef]

9. Liu, W.; Chau, K.T.; Lee, C.H.T.; Jiang, C.; Han, W. A Switched-Capacitorless Energy-Encrypted Transmitter for RoadwayCharging Electric Vehicles. IEEE Trans. Magn. 2018, 54, 1-6.

10. Dai, Z.; Fang, Z.; Huang, H.; He, Y.; Wang, J. Selective Omnidirectional Magnetic Resonant Coupling Wireless Power Transfer with Multiple-Receiver System. IEEE Access 2018, 6, 19287-19294. [CrossRef]

11. Huang, K.; Lau, V. Enabling Wireless Power Transfer in Cellular Networks: Architecture, Modeling and Deployment. IEEE Trans. Wirel. Commun. 2012, 13, 902-912. [CrossRef]

12. Sun, T.; Xie, X.; Li, G.; Gu, Y.; Deng, Y.; Wang, Z. A Two-Hop Wireless Power Transfer System with an Efficiency-Enhanced Power Receiver for Motion-Free Capsule Endoscopy Inspection. IEEE Trans. Biomed. Eng. 2012, 59, 3247-3254. [PubMed]

13. Hwang, S.-H.; Kang, C.G.; Lee, S.-M.; Lee, M.-Q. Reconfigurable Wireless Power Transfer System for Multiple Receivers. J. Electromagn. Eng. Sci. 2016, 16, 199-205. [CrossRef]

14. Madawala, U.K.; Thrimawithana, D.J. A bidirectional inductive power interface for electric vehicles in V2G systems. IEEE Trans. Ind. Electron. 2011, 58, 4789-4796. [CrossRef] 
15. Koh, K.E.; Beh, T.C.; Imura, T.; Hori, Y. Impedance Matching and Power Division Using Impedance Inverter for Wireless Power Transfer via Magnetic Resonant Coupling. IEEE Trans. Ind. Appl. 2014, 50, 2061-2070. [CrossRef]

16. Chen, L.J.; Boys, J.T.; Covic, G.A. Power Management for Multiple-Pickup IPT Systems in Materials Handling Applications. IEEE J. Emerg. Sel. Top. Power Electron. 2015, 3, 163-176. [CrossRef]

17. Zhang, Z.; Tong, R.; Liang, Z.; Liu, C.; Wang, J. Analysis and control of optimal power distribution for multi-objectivewireless charging systems. Energies 2018, 11, 1726. [CrossRef]

18. Kim, Y.-J.; Ha, D.; Chappell, W.J.; Irazoqui, P.P. Selective Wireless Power Transfer for Smart Power Distribution in a MiniatureSized Multiple-Receiver System. IEEE Trans. Ind. Electron. 2016, 63, 1853-1862. [CrossRef]

19. Liu, F.; Yang, Y.; Ding, Z.; Chen, X.; Kennel, R.M. A Multifrequency Superposition Methodology to Achieve High Efficiency and Targeted Power Distribution for a Multiload MCR WPT System. IEEE Trans. Actions Power Electron. 2018, 33, 9005-9016. [CrossRef]

20. Lee, K.; Cho, D.-H. Analysis of Wireless Power Transfer for Adjustable Power Distribution among Multiple Receivers. IEEE Antennas Wirel. Propag. Lett. 2015, 14, 950-953. [CrossRef]

21. Yin, H.; Fu, M.; Liu, M.; Song, J.; Ma, C. Autonomous Power Control in a Reconfigurable 6.78-MHz Multiple-Receiver Wireless Charging System. IEEE Trans. Ind. Electron. 2018, 65, 6177-6187. [CrossRef]

22. Fu, M.; Zhang, T.; Zhu, X.; Luk, P.; Ma, C. Compensation of cross coupling in multiple-receiver wireless power transfer systems. IEEE Trans. Ind. Inform. 2016, 12, 474-482. [CrossRef]

23. Zhen, N.L.; Chinga, R.A.; Tseng, R.; Lin, J. Design and test of a high-power high-efficiency loosely coupled planar wireless power transfer system. IEEE Trans. Ind. Electron. 2009, 56, 1801-1812. [CrossRef]

24. Shen, D.; Du, G.; Zeng, W.; Yang, Z.; Li, J. Research on Optimization of Compensation Topology Parameters for a Wireless Power Transmission System with Wide Coupling Coefficient Fluctuation. IEEE Access 2020, 8, 59648-59658. [CrossRef]

25. Roberts, M.J. Signals and Systems: Analysis Using Transform Methods \& MATLAB, 3rd ed.; McGraw-Hill Education: Boston, MA, USA, 2017.

26. Erickson, R.W. Fundamentals of Power Electronics; Kluwer Academic Publishers: New York, NY, USA, 2010.

27. Sedgewick, R.; Wayne, K. Algorithms, 4th ed.; Addison-Wesley Professional: Boston, MA, USA, 2011.

28. Fernandez, M. Models of Computation: An Introduction to Computability Theory; Springer: Berlin/Heidelberg, Germany, 2009. 